\title{
Respiratory monitoring using a Doppler radar with passive harmonic tags to reduce interference from environmental clutter
}

\author{
Aditya Singh, and Victor Lubecke, Senior Member, IEEE
}

\begin{abstract}
A harmonic tag was designed and fabricated to show the feasibility of sensing cardiopulmonary related Doppler shift in the received second harmonic signal. A fundamental frequency of $2.45 \mathrm{GHz}$ was transmitted at a target and a quadrature homodyne receiver used to sense the received signal from a tag on the target. The setup was used to successfully isolate periodic motion from a tag on a programmable mechanical target and to sense respiratory motion of a human subject, even when the targets were in close proximity to other moving objects.
\end{abstract}

\section{INTRODUCTION}

Harmonic tags can be used to isolate desired targets from clutter in Doppler radar systems. A system which can isolate small motion for a tagged target in the presence of motion of untagged objects would provide means for improved SNR for isolating cardiopulmonary motion from other body motion.

Harmonic tags have been successfully used earlier for uniquely separating a target from the environment [1], [2]. A harmonic tag consists of a tag antenna with a strongly non linear element at its port. In most cases, the non linear element is a schottky diode. The incoming signal is converted into harmonics by the non linear element and the tag is designed such that the second harmonic is transmitted back to the receiver. So far, the application of harmonic tags has been restricted to identification and tracking. By sensing their motion, the advantages of the harmonic tags can be applied to various applications such as cardiopulmonary monitoring and motion assessment.

\section{DOPPLER RADAR MOTION SENSING}

In the most simplistic case, the base-band output after mixing the received and the local oscillator signals is given by

\footnotetext{
Manuscript received April 7, 2009. This work was supported in part by the U.S. National Science Foundation under grant ECS0702234.

A. Singh and V. Lubecke are with the Department of Electrical Engineering, University of Hawaii at Manoa, Honolulu, HI 96822. (email:singha@ hawaii.edu)
}

$$
I_{B B}=A \cos \left[\frac{4 \pi f}{c} x(t)+\varphi_{\text {tot }}\right]
$$

where, $x(t)=h(t)+r(t)$, is the chest motion composed of heart motion $(\mathrm{h}(\mathrm{t}))$ and the respiration $(\mathrm{r}(\mathrm{t})) . \varphi_{\text {tot }}$ is the residual phase noise in the system. The theory behind cardiopulmonary monitoring using a Doppler radar has been well established [3], [4]. In summary, when a radiofrequency wave is incident on the body, the periodic motion of heart and breathing is converted into a phase shift in the reflected signal. This phase shift is detected and processed to show the vital signs of the human body. The basic schematic for the circuit used is shown in Fig. 1. The incoming RF signal is combined in a mixer to get the base band signal that contains the data.

On the complex I-Q plot, these equations form an arc that belongs to a circle centered at the origin.

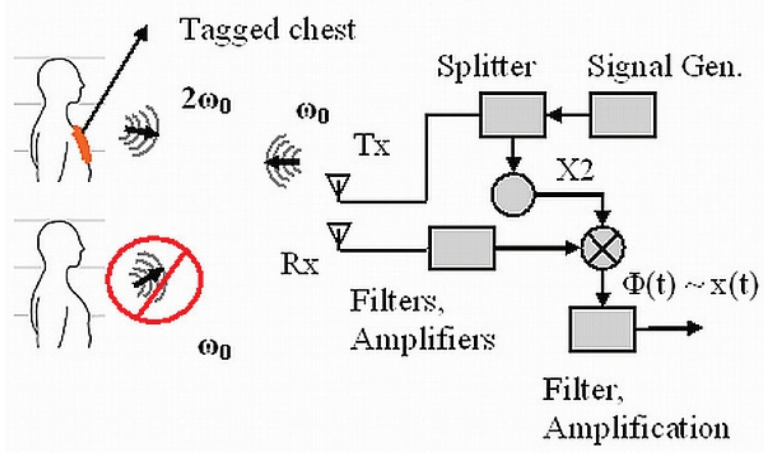

Fig.1..Proposed RF tagging for Doppler radar respiratory monitoring using harmonic tags.

\section{HARMONIC TAG}

A Harmonic tag was designed and fabricated to facilitate the sensing of human respiration through Doppler shift in a $4.9 \mathrm{GHz}$ harmonic backscatter signal.

RFID tag design theory was used to design the harmonic tag [5], [6]. The tag was designed and simulated using Agilent ADS momentum software. The tag was designed to keep its impedance very close to being the conjugate of 
the diode reactance at both $2.45 \mathrm{GHz}$ and 4.9 GHz. The network parameters were then exported to the schematic and simulated with diode. Thus both the harmonic performance and the overall scattering parameter of the tag could be evaluated.

The tag was constructed with a copper tape and placed over a $0.5 \mathrm{~cm}$ styrofoam substrate in order to avoid deleterious effect of the human body on the EM field of the antenna [7]. The tag was separately tested for its performance.

\section{EXPERIMENT SETUP}

The initial radar setup to sense the artificial motion is shown in Fig. 2(a). Two targets were moved at two different frequencies to distinguish the data from each other. The two targets consisted of the tag and a styrofoam ball having a diameter of an inch. The styrofoam ball was wrapped in aluminum foil to increase the scattering. The motion of the tag was very close to $0.3 \mathrm{~Hz}$ while the motion of the ball was at 1.3 $\mathrm{Hz}$. The distance between the targets and the antennas was 1 foot (Approximately 30 centimeters). The transmitted power to the antenna was $10 \mathrm{dBm}$. Five high pass filters and two RF amplifiers were used in the receiving circuit to remove the unwanted signals. The LO for the mixers was generated by splitting the transmitted signal from the signal generator and running it through a commercial frequency doubler. Two high pass filters and an amplifier were used to generate the LO input. Measurements were taken for three scenarios.

Case I - tag in motion/ball stationary

Case II - tag stationary/ball in motion

Case III - tag in motion/ball in motion

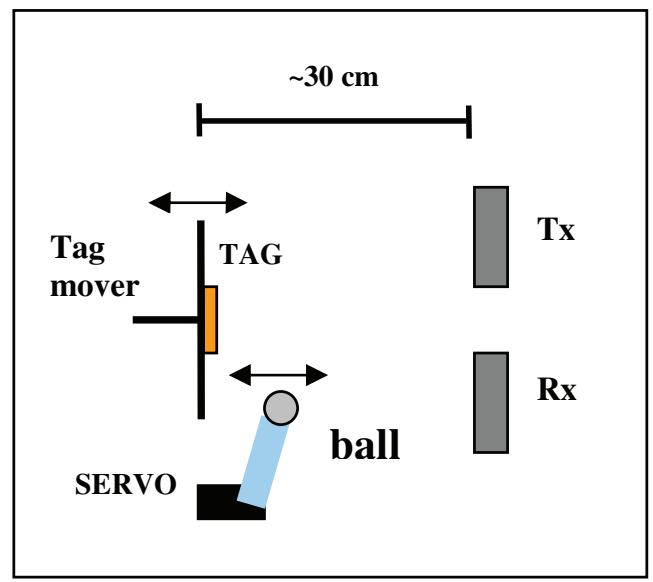

(a)

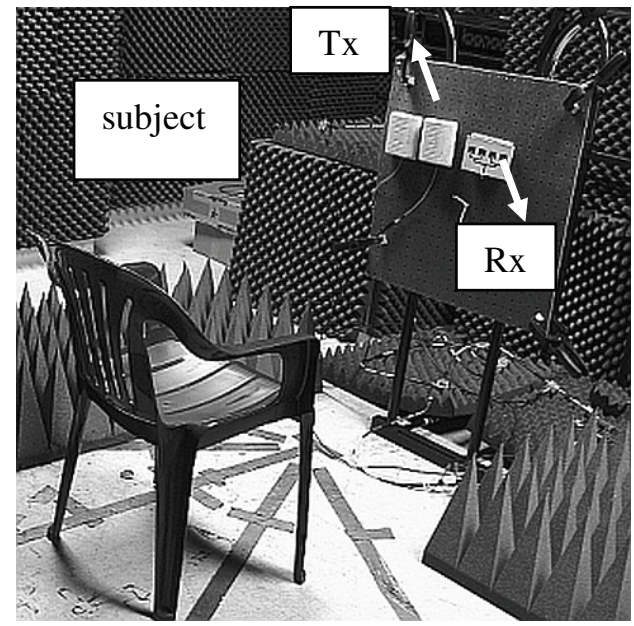

(b)

Fig.2.a) Schematic showing the measurement setup for mechanical targets and b) Photograph of the modified setup to carry out measurements on human subjects.

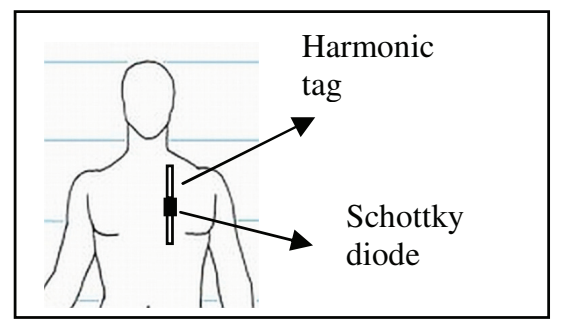

(a)

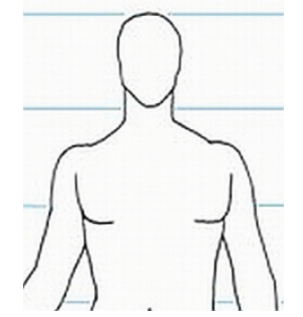

(b)

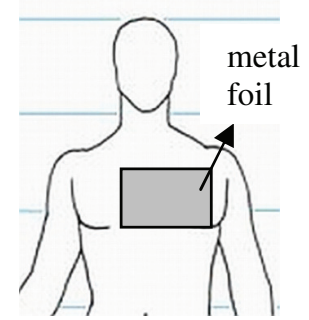

(c)
Fig.3. Three scenarios during human subject measurements, a) With the harmonic tag, b) Without the tag and c) With aluminum foil. Note that the Cross sectional area of the foil is more than the tag used. The subject was wearing a Tshirt at all times during the measurements and the tag and the foil were placed on the shirt.

The styrofoam ball was controlled by a servo which was controlled by a controller board. The movement of the Styrofoam ball was $1 \mathrm{~cm}$. The tag was placed in between the transmitting and receiving antenna while the ball was in front of the receiving antenna.

The setup was then modified such that human subjects could be measured conveniently. The modified setup is shown in Fig. 2(b). The tag was placed on the left side of the human chest. 
The distance between the tag and the antennas was approximately $30 \mathrm{~cm}$. A high pass filter and an $\mathrm{RF}$ amplifier were added in the receiving chain. The data was recorded using an A/D converter and MATLAB. The base-band signal was passed through a Low Noise Amplifier (LNA) before sending to the A/D converter. The sampling rate was selected to be $100 \mathrm{~Hz}$. Measurements were taken considering three scenarios as shown in Fig. 3.
a) Tag in front of Rx antenna
b) Without Tag
c) With a metal foil

The tag was attached to the human chest above the loose clothing.

\section{RESULTS}

The data obtained from the I channel was analyzed by means of fourier transform in order to retrieve the power level of the base-band signals. .

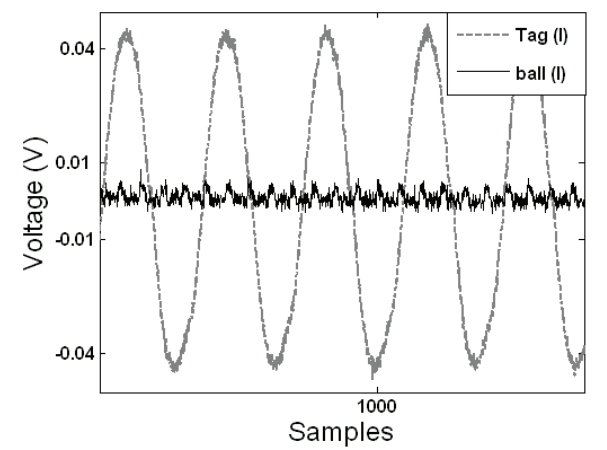

(a)

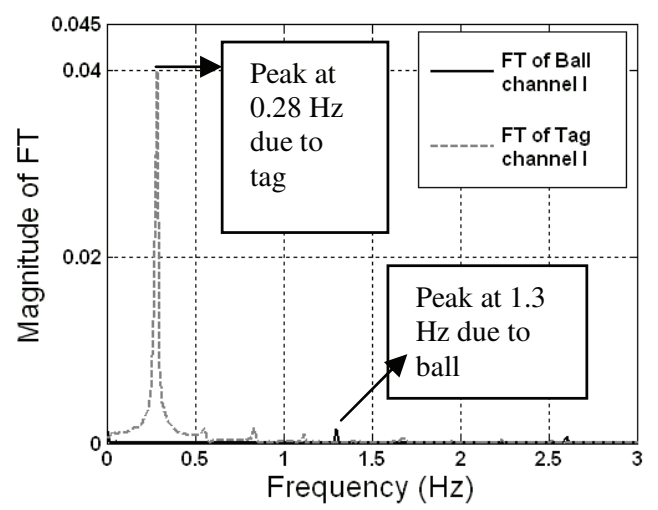

(b)

Fig.4. Combined data from case (I) and case (II), a) Voltage trace indicating the detected motion and b) fourier transform of the voltage trace showing the frequency of the detected motion hence indicating the ability of the radar to detect the tag in case (I) and reject the signal due to ball in case(II).

Fig. 4 shows the measured data and the fourier transform of case (I) and case (II). Fig. 4(a) compares the voltage signal from the channel I for aforementioned cases. The data has been displayed for 1500 samples (15 seconds) in order to clearly represent the signals. However, the fourier transform was performed on the entire dataset. It can be inferred from the figure that the voltage detected due to the motion of the tag alone is significantly higher than the voltage detected due to the motion of the Styrofoam ball. The fourier transform in Fig. 4 (b) also shows that the ratio of $0.3 \mathrm{~Hz}$ signal to $1.3 \mathrm{~Hz}$ signal is approximately $28: 1$.

Fig. 5 displays the data for the case (III) when both the targets were moving simultaneously. The presence of $1.3 \mathrm{~Hz}$ can be seen in the voltage trace and the fourier transform

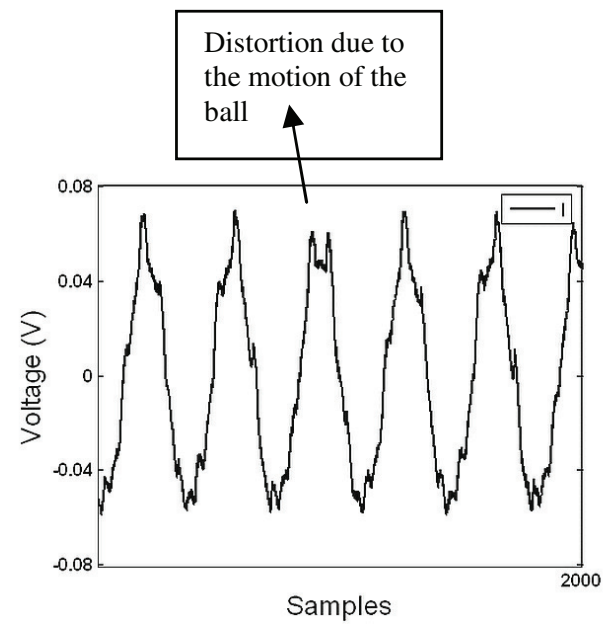

(a)

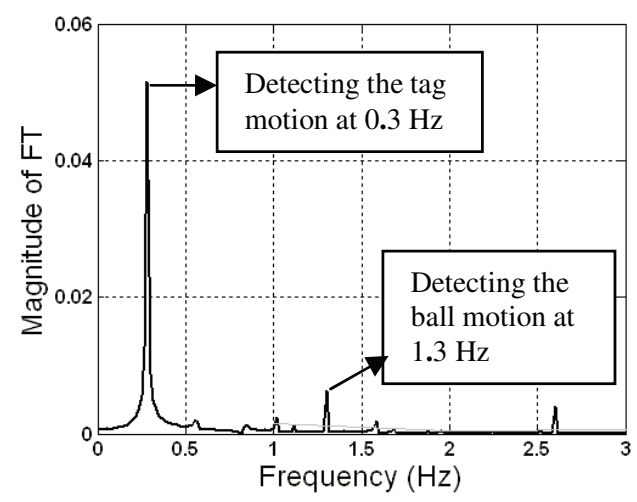

(b)

Fig.5. Data from case (III), a) Voltage trace indicating very little distortion due to the styrofoam ball and b) Fourier transform of the data. The ratio of the magnitude of the signals due to tag and the ball is approximately 9:1. 
However, the detected component at $1.3 \mathrm{~Hz}$ in case III is a little higher compared to that in case II. The data from Fig 5(b) illustrates the ability of the harmonic radar to reject the Doppler shift due to the fundamental frequency component.

The data and fourier transform for tests on a human subject for the three scenarios is displayed in the figures below. There is some DC drift observed which might be due to slight movement of the torso or due to the LNA's.

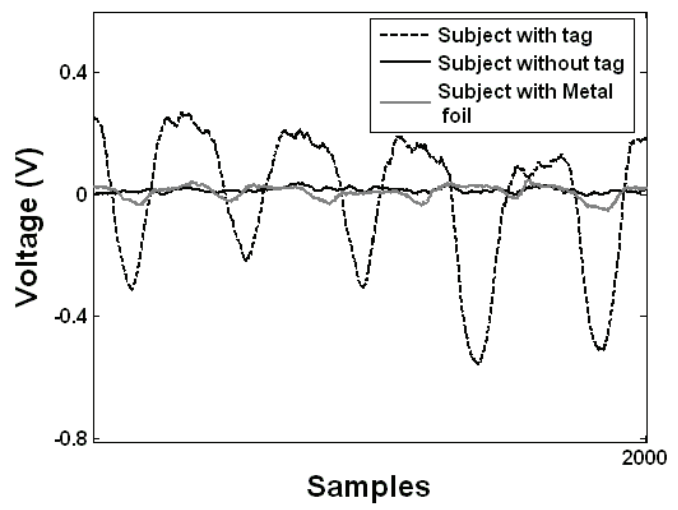

(a)

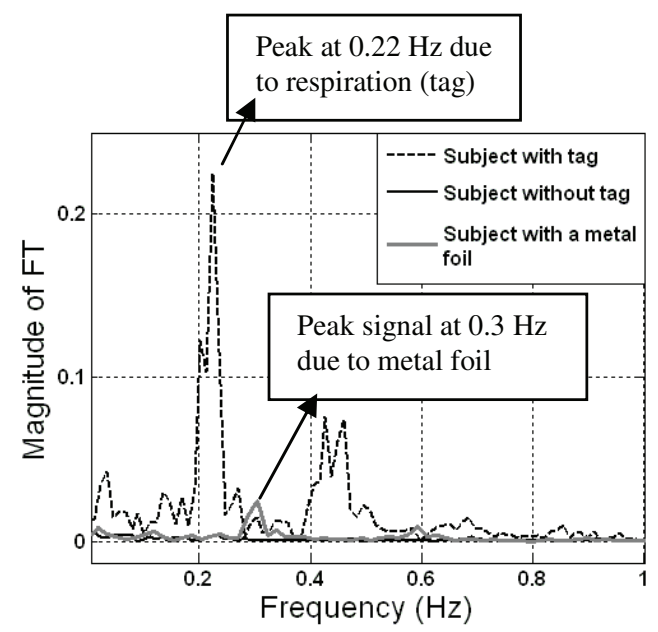

(b)

Fig.6. Combined data from the scenarios (a), (b) and (c) a) data from A/D converter and b) Fourier transform of the voltage trace showing the detected respiration signal with the harmonic tag and the low magnitude signal detected due to metal foil.

Fig. 6 (a) compares the voltage signal obtained from the three scenarios. The signal obtained when the human subject is wearing the tag is much larger than the other scenarios. The FFT of the data also indicates this in Fig.6 (b). The ratio of the strongest detected signal with tag to the strongest detected signal with metal foil is 9.2 whereas the data obtained without the tag is buried in noise.

\section{CONCLUSION}

The harmonic radar setup was successfully used to detect the tag and reject any other motion by a suitably sized object. The harmonic radar also isolated the respiration of a human subject from all the other motion that is associated with the respiration. In addition to that, the radar was also shown to effectively suppress the doppler shift due to the metal foil at the fundamental frequency. If a narrowband low noise RF amplifier is used in the receiver to amplify the $4.9 \mathrm{GHz}$ signal, the performance of the system is further expected to improve along with the range. An improved system could be effectively used to reject the signal due to any other untagged body motion while detecting respiration of the tagged chest. Such a system could be used to continuously monitor a patient alerting the medics in case of abnormalities.

\section{ACKNOWLEDGMENT}

The author would like to thank J. Kiriazi for his support during the course of experiments and Isar Mostafanezjad and N. Hafner for their assistance in taking measurements.

\section{REFERENCES}

[1]. Bruce G. Colpitts and Gilles Boiteau, "Harmonic Radar Transceiver Design: iniature tags for insect tracking", IEEE Transactions on Antennas and Propagation, Vol. 52, No. 11, Nov 2004.

[2]. John Kiriazi, Jayson Nakamura, Kevin Hall and Victor Lubecke, "Low profile Harmonic Radar Transponder for tracking small endangered species", Engineering in Medicine and Biology Society, 2007. EMBS 2007. 29th Annual International Conference of the IEEE. Volume, Issue, 22-26 Aug. 2007, pp. 2338 - 2341.

[3]. Amy Droitcour, Victor Lubecke, Jenshan Lin and Olga Boric-Lubecke, "A Microwave Radio for Doppler Radar Sensing of Vital Signs, "2001 IEEE MTT-S Digest, Vol.1 pp. 175-178.

[4]. Victor Lubecke, Olga Boric-Lubecke, Eric Beck, "A compact low cost add on module for Doppler Radar Sensing of Vital Signs using a wireless communications terminal", 2002 IEEE MTT-S Digest, Vol.3, pp. 1767-1770.

[5]. K. V. Seshagiri Rao, Pavel V. Nikitin and Sander F. Lam, "Antenna Design for UHF RFID Tags: A review and a practical application", IEEE Transactions on Antennas and Propagation, Vol. 53, No. 12, Dec 2005

[6]. Daniel M. Dobkin, "The RF in RFID: Passive UHF RFID in Practice". Publisher Newnes.

[7]. M Sanad, "Effect of human body on microstrip antennas", Antennas and propagation society international symposium, 1994. AP-S Digest, Vol. 1, pp. 298-301. 